\title{
Suscetibilidade à podridão negra e produtividade de brócolis em função de doses de nitrogênio e potássio
}

\author{
Santino Seabra Junior'; Juliana G de Lalla²; Rumy Goto²; Antonio C Maringoni²; Roberto L Villas Boas ${ }^{2}$; \\ Janaina RC Rouws ${ }^{3}$; Eduardo E Oriani² \\ ${ }^{1}$ UNEMAT, Depto. Agronomia, Av. São João s/nº $78200-000$ Cáceres-MT; santinoseabra@hotmail.com; ${ }^{2}$ UNESP-FCA, Depto Produção \\ Vegetal, C. Postal 237, 18610-307 Botucatu-SP; jugadum@hotmail.com; rumy@fca.unesp.br; maringoni@fca.unesp.br; rlvboas@fca. \\ unesp.br; duoriani@hotmail.com; ${ }^{3}$ Embrapa Agrobiologia, C. Postal 74505, 23890-000 Seropédica-RJ; janaina.rouws@embrapa.br
}

\section{RESUMO}

O objetivo do presente estudo foi investigar a influência da adubação com nitrogênio e com potássio na severidade da podridão negra e na produtividade de brócolis tipo inflorescência única. $\mathrm{O}$ delineamento utilizado foi em blocos casualizados, com 4 repetições, em esquema de parcelas subdivididas com fatorial nas subparcelas. Na parcela avaliou-se a influência da inoculação (plantas injuriadas com água destilada e inoculadas com Xanthomonas campestris pv. campestris) e na subparcela avaliou-se o fatorial $4 \times 4$ correspondendo a doses de potássio $\left(0,275,550\right.$ e $\left.825 \mathrm{~kg} \mathrm{ha}^{-1}\right)$ e de nitrogênio $(0,150$, 300 e $\left.450 \mathrm{~kg} \mathrm{ha}^{-1}\right)$. O brócolis híbrido BRO68 foi inoculado utilizando o método de palito aos 64 dias após a semeadura. Foi verificado que maiores doses de potássio aplicadas reduziram a severidade da doença, porém a ausência ou o excesso de nitrogênio aumentaram a severidade da doença. Estimou-se que as maiores produções de inflorescência de brócolis foram obtidas nas doses de 541 a $751 \mathrm{~kg}$ $\mathrm{ha}^{-1}$ de $\mathrm{K}_{2} \mathrm{O}$ e 286 a $328 \mathrm{~kg} \mathrm{ha}^{-1}$ de N. No entanto, ao se considerar uma adubação equilibrada para obter maior produtividade, tamanho de inflorescência e maior resistência à podridão negra as doses indicadas são de $283 \mathrm{~kg} \mathrm{ha}^{-1}$ de $\mathrm{N}$ e $550 \mathrm{~kg} \mathrm{ha}^{-1}$ de $\mathrm{K}_{2} \mathrm{O}$.

Palavras-chave: Brassica oleracea var. italica, Xanthomonas campestris pv. campestris, doença, adubação, nutrição.

\begin{abstract}
Susceptibility to black rot and yield of broccoli depending on nitrogen and potassium doses

The purpose of this study was to investigate the influence of potassium and nitrogen on the severity of black rot and yield of head. The experiment was carried out in split plots with factorial subplots, in randomized blocks with four replications. In the plots we assessed the influence of inoculation (injured plants with distilled water and inoculated with Xanthomonas campestris pv. campestris) and in the subplots we evaluated the $4 \times 4$ factorial, potassium doses $(0,275$, 550 and $\left.825 \mathrm{~kg} \mathrm{ha}^{-1}\right)$ and nitrogen $\left(0,150,300\right.$ and $\left.450 \mathrm{~kg} \mathrm{ha}^{-1}\right)$. The hybrid Broccoli BRO68 was inoculated using the toothpick method at 64 days after sowing. We observed that potassium in the plant reduced the disease, but nitrogen increased disease. We estimated that the highest levels of potassum in the plant reduced the severity of the disease but lack or excess of nitrogen increased disease severity. The highest production of broccoli inflorescence was obtained at doses from 541 to $751 \mathrm{~kg} \mathrm{ha}^{-1}$ of $\mathrm{K}_{2} \mathrm{O}$ and 286 to $328 \mathrm{~kg} \mathrm{ha}^{-1}$ of N. However, when considering a balanced fertilization to obtain higher yield and size of inflorescences and greater resistance to black rot, the ideal doses are $283 \mathrm{~kg} \mathrm{ha}^{-1}$ of $\mathrm{N}$ and $550 \mathrm{~kg} \mathrm{ha}^{-1}$ of $\mathrm{K}_{2} \mathrm{O}$.
\end{abstract}

Keywords: Xanthomonas campestris pv. campestris, Brassica oleracea var. italica, disease, fertilization, nutrition.

(Recebido para publicação em 26 de abril de 2012; aceito em 24 de julho de 2013) (Received on April 26, 2012; accepted on July 24, 2013)

$\mathrm{O}$ brócolis (Brassica oleracea var. italica) é uma planta exigente em nitrogênio e potássio (Rincon et al., 1999). Na literatura encontram-se estudos em que foram obtidas as produtividades máximas com doses de nitrogênio variando de 196 a $558 \mathrm{~kg}$ ha $^{-1}$ (Kowalenko \& Hall, 1987; Kahn et al., 1991; Everaarts, 1994; Zebarth et al., 1995; Everaarts \& Willigen, 1999) e dose de potássio de até $643,2 \mathrm{~kg} \mathrm{ha}^{-1}$ de $\mathrm{K}_{2} \mathrm{O}$ (Peck \& Macdonald, 1986). No estado de São Paulo, as doses recomendadas variam de 75 a $260 \mathrm{~kg} \mathrm{ha}^{-1}$ para nitrogênio e 180 a $360 \mathrm{~kg} \mathrm{ha}^{-1}$ de $\mathrm{K}_{2} \mathrm{O}$ para potássio (Trani \& Raij, 1997). Ava- liando o potencial produtivo da espécie e verificando que as doses recomendadas são menores que as relatadas anteriormente, justifica-se esse estudo.

Outro fator importante a ser considerado é a influência destes nutrientes na suscetibilidade à doença. Zambolim \& Ventura (1996) citam que a deficiência ou o excesso de um elemento influencia a atividade de outros e exerce efeito notável com conseqüências que repercutem no metabolismo, na morfologia e na anatomia das plantas induzindo maior tolerância ou suscetibilidade à doença destas plantas no campo (Huber, 1994).

A adubação nitrogenada e a potás- sica têm importância destacada, tanto na produtividade quanto na resistência a doenças (Huber, 1994; Marschner, 1995; Silveira \& Higashi, 2003; Yamada, 2005). Sendo que o efeito do nitrogênio na resistência de doenças é totalmente dependente da dose, pois em doses adequadas contribui para a síntese de fenóis e alcalóides, mas em altas concentrações reduz a produção de compostos fenólicos (Yamada, 2004) e também resulta na produção de tecidos jovens e suculentos, podendo prolongar o estádio vegetativo, criando condições favoráveis ao ataque de patógenos. O potássio por sua vez, auxilia na resis- 
tência a doenças, podendo equilibrar a adubação nitrogenada e tornar os tecidos mais fibrosos, aumentando a resistência a fungos e bactérias (Huber, 1994; Filgueira, 2008; Yamada, 2005).

Estudos relacionando nutrição $\mathrm{x}$ doença relatam que altas doses de nitrogênio na adubação, podem aumentar a incidência e a severidade de diversas doenças como podridão mole causada por Erwinia carotovora subsp. carotovora (Everaarts, 1994) e podridão negra causada por Xanthomonas campestris pv. campestris (Silva Junior, 1986) porém, o último autor afirma que em repolho este efeito deletério do nitrogênio foi amenizado com a presença da adubação potássica.

A podridão negra vem promovendo consideráveis reduções de produtividade e qualidade em brássicas (Seabra Júnior et al, 2008), áreas de produção de brócolis vem apresentando alta incidência e severidade desta doença, em diferentes regiões do país. Os sintomas da podridão negra manifestam-se em qualquer fase de desenvolvimento das plantas e são transmitidas por sementes, nas folhas caracterizam-se por lesões amarelas em forma de "V", com o vértice voltado para o centro da folha. Com o desenvolvimento da doença, as folhas tornam-se amarelas e podem apresentar necrose. Em alguns casos, observa se subdesenvolvimento, murcha, queda prematura de folhas e apodrecimento das plantas afetadas (Peruch et al., 2006).

O objetivo do presente estudo foi investigar a influência de doses de nitrogênio e de potássio na severidade da podridão negra e na produtividade de brócolis tipo inflorescência única.

\section{MATERIAL E MÉTODOS}

O experimento foi conduzido no Departamento de Produção Vegetal da UNESP em Botucatu (22 $52^{\prime} 20^{\prime}$ 'S, 48 26'37' O, altitude média $800 \mathrm{~m}$ ). O cultivo do brócolis foi realizado sob ambiente protegido no período entre fevereiro e junho de 2004. As médias das temperaturas mínimas e máximas foram de 15,7 e $31,6^{\circ} \mathrm{C}$, respectivamente.

$\mathrm{O}$ delineamento experimental utilizado foi de blocos casualizados, com 4 repetições, em esquema de parcelas sub- divididas com fatorial nas subparcelas, avaliando-se nas parcelas as inoculações, em que as plantas foram injuriadas com palito de madeira umedecido em água destilada (testemunha) e com inóculo de Xanthomonas campestris pv. campestris (isolado patogênico CM2917A). Nas subparcelas foi utilizado o esquema fatorial $4 \times 4$, avaliando quatro doses de potássio $(0,275,550$ e $\left.825 \mathrm{~kg} \mathrm{ha}^{-1}\right)$ e quatro doses de nitrogênio $\left(0,150,300\right.$ e $\left.450 \mathrm{~kg} \mathrm{ha}^{-1}\right)$.

Neste estudo, foi utilizado o brócolis tipo inflorescência única, híbrido BR068, por apresentar suscetibilidade à podridão negra (Seabra Júnior et al., 2008) e apresentar maior potencial produtivo em condições de clima tropical (Lalla et al, 2010).

As mudas foram produzidas em bandejas de poliestireno expandido 128/6 preenchidas com substrato comercial, as quais foram transplantadas aos 33 dias após semeadura (apresentando quatro folhas definitivas) em vasos contendo $10 \mathrm{~L}$ de solo, com uma planta por vaso e cinco plantas por subparcela.

O solo utilizado foi classificado como Latossolo Vermelho Escuro Álico, textura média. Depois de corrigido com calcário dolomítico e realizada a adubação de plantio com fósforo [superfosfato simples $\left(18 \%\right.$ de $\left.\left.\mathrm{P}_{2} \mathrm{O}_{5}\right)\right] \mathrm{o}$ solo apresentou em média as seguintes características químicas: $\mathrm{M} . \mathrm{O}=8,0 \mathrm{~g}$ $\mathrm{dm}^{3} ; \mathrm{pH}=7,0, \mathrm{P}=41,70 \mathrm{mg} \mathrm{dm}{ }^{-3} ; \mathrm{Ca}=$ 46,7 $\mathrm{mmol}_{\mathrm{c}} \mathrm{dm}^{-3} ; \mathrm{Mg}=7,7 \mathrm{mmol}_{\mathrm{c}} \mathrm{dm}^{-3}$; $\mathrm{CTC}=65,7 ; \mathrm{V}=85,5 \%$. Após a adubação de plantio com potássio com $45 \%$ da dose correspondente a cada tratamento estudado, utilizando cloreto de potássio ( $60 \%$ de $\mathrm{K}_{2} \mathrm{O}$ ), verificou-se no solo os teores de $0,4,1,1,1,6$ e 2,5 $\mathrm{mmol}_{\mathrm{c}} \mathrm{dm}^{-3}$, respectivamente para os tratamentos de $0,275,550$ e $825 \mathrm{~kg} \mathrm{ha}^{-1}$ de $\mathrm{K}_{2} \mathrm{O}$. Na adubação de plantio também foi aplicado $10 \%$ do nitrogênio [uréia (45\% de N)] conforme os tratamentos para adubação nitrogenada.

A adubação de cobertura foi realizada no sistema de fertirrigação aplicando-se os adubos potássicos e nitrogenados conforme a dose correspondente ao tratamento, parcelando em oito épocas (dias após o transplante, DAT), sendo: aos 6 e 10 DAT $(5 \%$ N), 14 e 22 DAT $\left(12,5 \% \mathrm{~N}\right.$ e $\left.5 \% \mathrm{~K}_{2} \mathrm{O}\right), 37$ e 43 DAT $\left(12,5 \% \mathrm{~N}\right.$ e $\left.10 \% \mathrm{~K}_{2} \mathrm{O}\right), 48$ DAT $(15 \%$ $\mathrm{N}$ e $\left.10 \% \mathrm{~K}_{2} \mathrm{O}\right)$ e 55 DAT $(15 \% \mathrm{~N}$ e $15 \%$ $\mathrm{K}_{2} \mathrm{O}$ ), utilizando como fonte a uréia e o cloreto de potássio. Na primeira adubação de cobertura foi inserido $5,0 \mathrm{~kg} \mathrm{ha}^{-1}$ de bórax (11\% B), $14 \mathrm{~kg} \mathrm{ha}^{-1}$ de sulfato de zinco $(22 \% \mathrm{Zn}), 37 \mathrm{~kg} \mathrm{ha}^{-1}$ de gesso agrícola $(15 \% \mathrm{~S})$. Também foi realizada a adubação foliar com bórax (11\% B) na concentração de $1 \mathrm{~g} \mathrm{~L}^{-1}$ e molibdato de amônio (54\% Mo) na concentração de $0,5 \mathrm{~g} \mathrm{~L}^{-1}$ via foliar aos 15 e 30 dias após o transplante.

O espaçamento utilizado foi de $0,6 \mathrm{x}$ $0,5 \mathrm{~m}$ entre plantas. $\mathrm{O}$ sistema de irrigação foi de gotejamento, com mangueiras com espaçamento entre os emissores de $0,5 \mathrm{~m}$ sob os vasos, sendo realizada uma vez ao dia e suplementado manualmente para atender a necessidade da cultura.

Para a inoculação foi utilizado o isolado CM2917A, obtido de plantas de couve-flor, avaliado como mais patogênico. As plantas foram inoculadas com 6 a 8 folhas definitivas (27 dias após transplante), pelo método de 'palito', que consiste em introduzir o palito de dente previamente embebido por três horas no inóculo ou em água no caule da planta, num local eqüidistante entre as folhas cotiledonares e a primeira folha (Henz et al., 1988).

Para preparo do inóculo utilizou-se colônias puras do isolado repicadas para placas de Petri, contendo meio de cultura NSA, e incubadas a $28^{\circ} \mathrm{C}$, durante $72 \mathrm{~h}$.

A concentração de $10^{8}$ UFC $\mathrm{mL}^{-1}$ foi obtida a partir de diluições feitas de acordo com a curva de calibração previamente obtida ( $\mathrm{Y}=0,9022-0,7665 \mathrm{X}$; onde $\mathrm{Y}=$ absorbância e $\mathrm{X}=\mathrm{UFC} \mathrm{mL}^{-1}$ $10^{8}$ ), ajustadas em espectrofotômetro, no comprimento de onda de $550 \mathrm{~nm}$.

A colheita iniciou-se aos 99 dias após a semeadura e encerrou-se aos 114 dias, coletando-se toda a parte aérea, onde foram verificados o número médio de nós (NN) e o número de folhas (FP) presentes nas plantas, com os quais se calculou a freqüência relativa das folhas retidas na planta (FR), através da fórmula $\mathrm{FR}=(\mathrm{FP} \times 100) / \mathrm{NN}$; além da porcentagem da área necrosada do caule (PANC), adaptada de Henz et al. (1988), obtida através da secção longitudinal do caule, medindo o comprimento da ne- 
crose interna (CNI) e o comprimento do caule $(\mathrm{CC})$, calculada através da fórmula $\mathrm{PANC}=(\mathrm{CNI} \times 100) / \mathrm{CC}$. Para análise estatística desta variável foi considerado o esquema fatorial $4 \times 4$ por ser possível avaliar dados provenientes somente da inoculação com $X$. campestris pv. campestris. Para mensurar a produtividade $\left(\mathrm{g}\right.$ planta $\left.{ }^{-1}\right)$, foram colhidas inflorescências cortando-se na base da primeira folha, no momento em que a inflorescência atingia o máximo crescimento, mas apresentavam-se compactas e com os grânulos bem fechados.

Para análise dos dados foi utilizado o programa SISVAR (Ferreira, 2000). Estes foram submetidos à análise de variância e, aos resultados significativos pelo teste $\mathrm{F}$ a $5 \%$ de probabilidade e à análise de regressão polinomial a $5 \%$ de probabilidade, respectivamente quando o fator era qualitativo e quantitativo. As médias referentes às doses de nitrogênio e potássio foram ajustadas em equações de regressão e apresentadas em forma de gráficos somente quando estas foram significativas ao nível de 5\% de probabilidade pelo teste $\mathrm{F}$.

\section{RESULTADOS E DISCUSSÃO}

Considerando-se as variáveis estu-

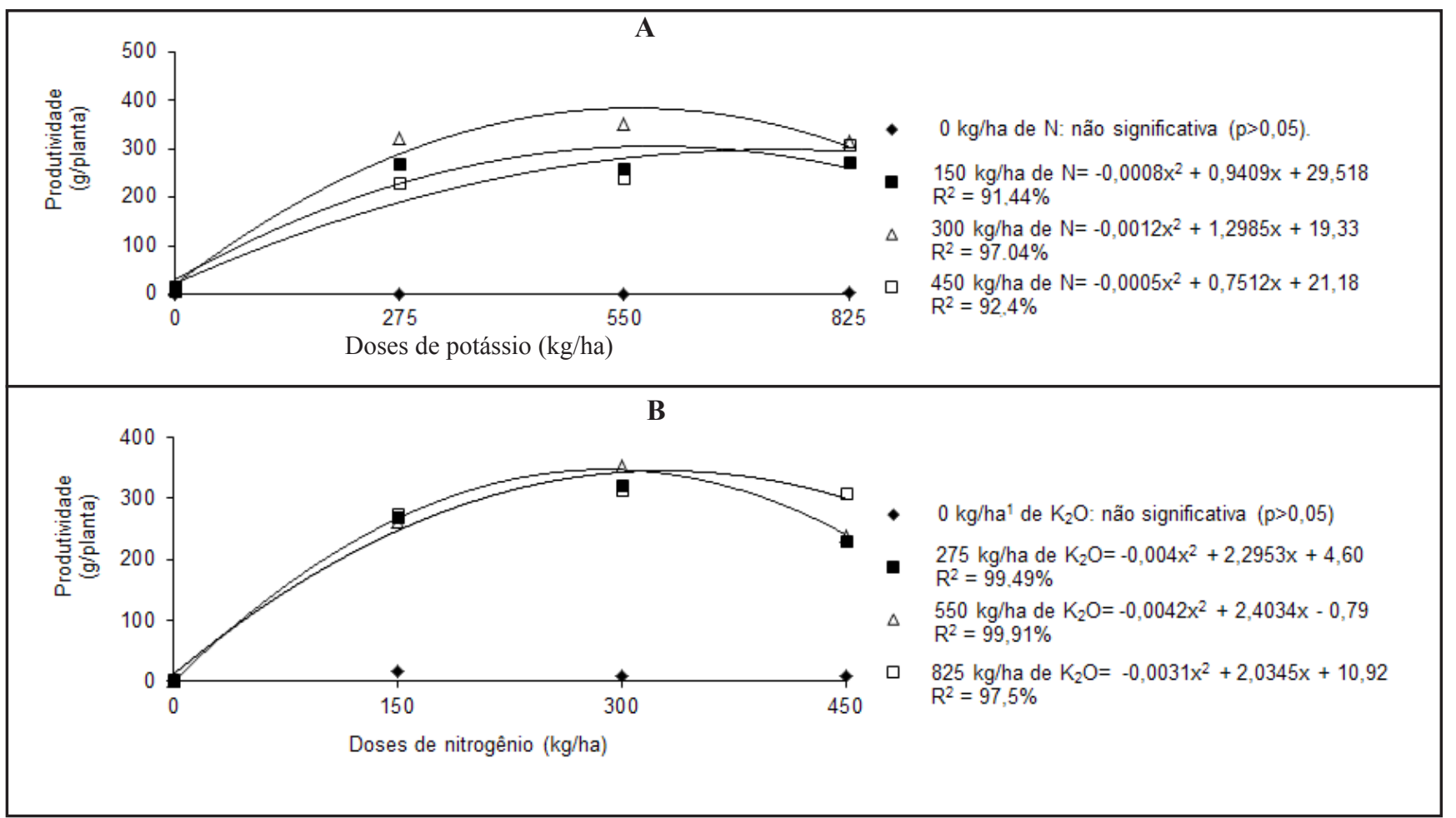

Figura 1. Produtividade de brócolis tipo cabeça única em função de doses de potássio dentro de cada dose de nitrogênio (A) e de nitrogênio dentro de cada dose de potássio (B) [yield of head broccoli, depending on potassium levels within each dose of nitrogen (A) and nitrogen within each dose of potassium (B)]. Botucatu, UNESP, 2004.

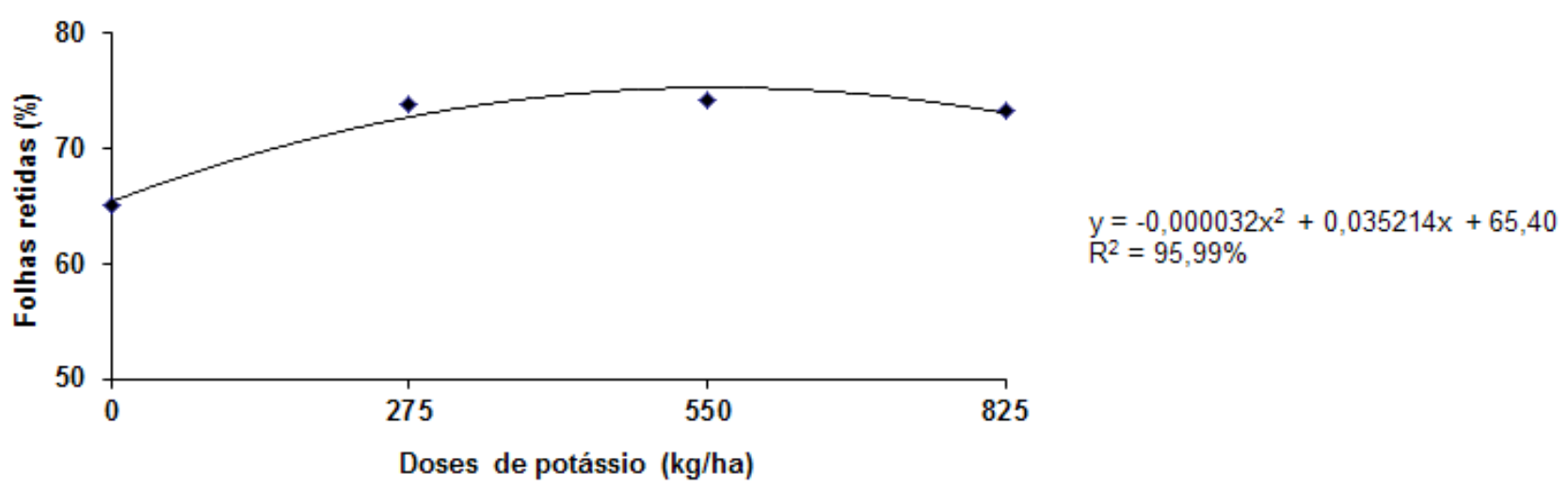

Figura 2. Folhas retidas de brócolis tipo cabeça única (\%) em função de doses de potássio (retained leaves (\%) of head broccoli, depending on potassium doses). Botucatu, UNESP, 2004. 


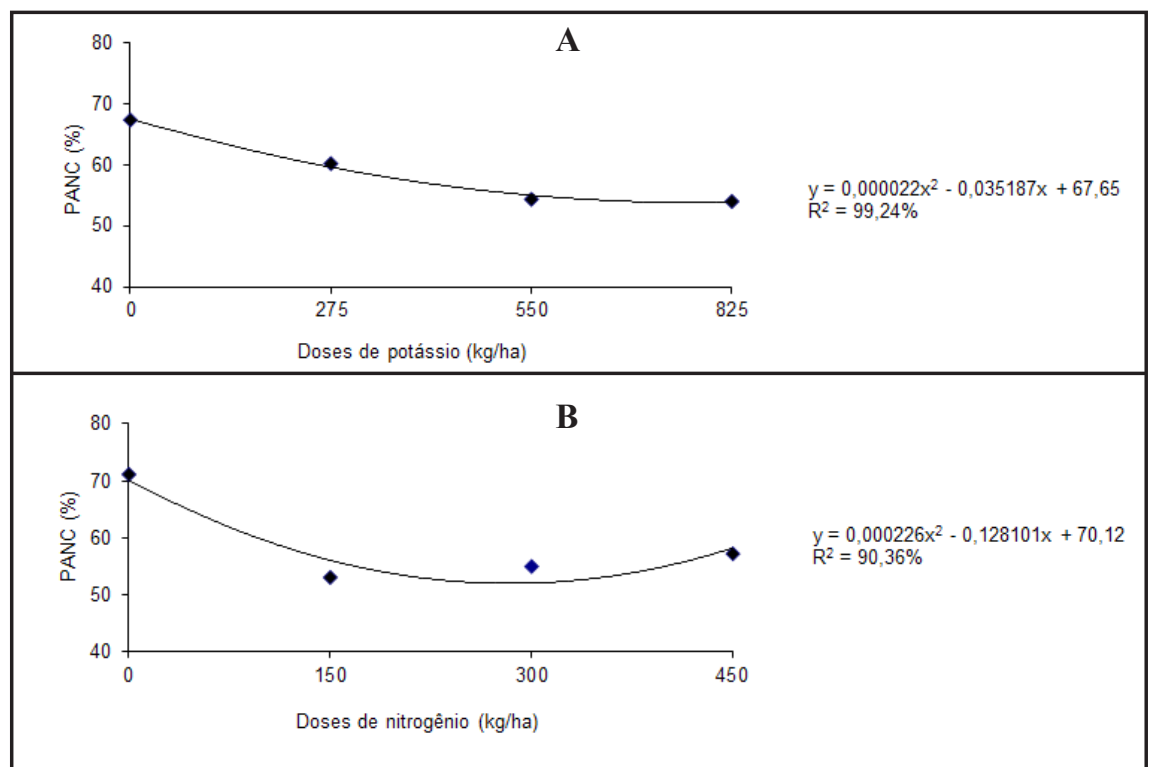

Figura 3. Área necrosada (\%) do caule (PANC) de brócolis tipo cabeça única em função de doses de potássio (A) e nitrogênio (B) [necrotic área (\%) of the stem (PANC) of head broccoli, depending on potassium (A) and nitrogen (B) doses]. Botucatu, UNESP, 2004.

Tabela 1. Produtividade e folhas retidas de brócolis (\%), com e sem inoculação com Xanthomonas campestris pv. campestris (yield and retained leaves of broccoli (\%) inoculated or not with Xanthomonas campestris pv. campestris). Botucatu, UNESP, 2004.

\begin{tabular}{lcc}
\hline Tratamentos & Produtividade (g/planta) & Folhas retidas (\%) \\
\hline Não inoculado & $173,50 \mathrm{a}$ & $77,40 \mathrm{a}$ \\
Inoculado & $152,35 \mathrm{~b}$ & $65,77 \mathrm{~b}$ \\
\hline
\end{tabular}

Médias seguidas de letras distintas na coluna, diferem entre si pelo teste $F(p<0,05)$ [means followed by different letters in the column, are different through $F$ test $(\mathrm{p}<0.05)]$.

dadas foi observada interação significativa para adubação com potássio $\mathrm{x}$ nitrogênio apenas para produtividade de inflorescência, sendo o fator inoculação estudado de forma separada.

Para a característica porcentagem da área necrosada do caule (PANC), somente as plantas inoculadas com $X$. campestris pv. campestris apresentaram sintomas de necrose interna do caule, como esperado.

Foram observadas diferenças significativas entre as plantas controle e as plantas inoculadas com $X$. campestris pv. campestris para a produtividade. Para o controle foi observado uma superioridade na produtividade de $13 \%$. As médias de produções entre os tratamentos encontradas foram de 173,5 e 152,35 g planta $^{-1}$, respectivamente, para as plantas controle e inoculadas (Tabela 1). Essa redução da produtividade foi causada pela colonização vascular da bactéria nos vasos de xilema e pela que- população de plantas superior a 33.333 plantas ha ${ }^{-1}$ utilizada nesse trabalho. Porém, ao comparar com os dados de Lalla et al. (2010) que obtiveram uma produtividade de 770,00 $\mathrm{g} \mathrm{planta}^{-1}$ numa densidade de 20.000 plantas por ha- ${ }^{-1}$, a produtividade obtida nesse trabalho foi inferior. $\mathrm{O}$ efeito do espaçamento sobre a produtividade de brócolis foi observado por Schiavon Júnior (2008), que obteve maior produtividade de inflorescência em plantas cultivadas em espaçamentos maiores. Foi verificado que nos tratamentos onde não foram adubados com nitrogênio (Figura 1A) ou potássio (Figura 1B) não houve resposta significativa para o aumento das doses de adubo estudadas, sendo que a produtividade de inflorescência foi insignificante, obtendo médias de 0,3 a 16,4 g planta $^{-1}$ de inflorescência. Durante a condução do experimento foram observadas plantas com severos sintomas de deficiência de nitrogênio, as quais apresentaram porte reduzido, caules e folhas arroxeados, e quando se tornavam mais velhas, ficavam vermelho-amareladas. A emissão da inflorescência foi mais tardia, e a planta produziu inflorescência pequena de 2 $\mathrm{cm}$ de diâmetro. Estas foram colhidas no último dia de colheita (114 dias após semeadura), ao supor que estas não apresentariam maior desenvolvimento. Os sintomas foliares descritos foram semelhantes aos observados por Homa et al. (1981) em couve flor.

Nas plantas que não receberam adubação potássica, os sintomas foram o pequeno desenvolvimento das plantas, as folhas se apresentaram rugosas e arqueadas para baixo, com queimas marginais, sintoma típico de deficiência de potássio, além de a inflorescência ser pouco compacta. Esses sintomas nas folhas foram semelhantes àqueles descritos por Homa et al. (1981) para couve-flor.

Na presença da adubação nitrogenada $\left(150,300\right.$ e $\left.450 \mathrm{~kg} \mathrm{ha}^{-1} \mathrm{~N}\right)$, quando foram avaliadas doses crescentes de potássio para cada dose de nitrogênio $\left(150,300\right.$ e $\left.450 \mathrm{~kg} \mathrm{ha}^{-1}\right)$ foram obtidos efeitos quadráticos para a produtividade (Figura 1A). As doses que proporcionaram produções máximas estimadas de $306,17,370,61$ e 303,33 g planta $^{-1}$ de 
inflorescência foram 588,06, 541,04 e $751,20 \mathrm{~kg} \mathrm{ha}^{-1}$ de $\mathrm{K}_{2} \mathrm{O}$, respectivamente quando se utilizou 150,300 e $450 \mathrm{~kg}$ $\mathrm{ha}^{-1}$ de N.

As doses estimadas neste experimento são praticamente o dobro das recomendadas por Trani \& Raij (1997), que variam de 180 a $360 \mathrm{~kg} \mathrm{ha}^{-1} \mathrm{de}_{2} \mathrm{O}$, mas se aproximam dos $643,2 \mathrm{~kg} \mathrm{ha}^{-1} \mathrm{de}$ $\mathrm{K}_{2} \mathrm{O}$ avaliados por Peck \& MacDonald (1986), e se justifica pela alta extração de potássio obtido por Magnífico et al. (1979) e Rincon et al. (1999), 723 e 261 $\mathrm{kg} \mathrm{ha}^{-1}$ de potássio, respectivamente. Schiavon Júnior (2008), num estudo comparando doses de nitrogênio e potássio e espaçamento observou maior produtividade de inflorescência utilizando as doses de 233 e $315 \mathrm{~kg} \mathrm{ha}^{-1}$ de $\mathrm{K}_{2} \mathrm{O}$ e $\mathrm{N}$, dependendo da densidade de plantas. Porém, este estudo foi realizado em um solo com alto teor de potássio (4,9 $\left.\mathrm{mmol}_{\mathrm{c}} \mathrm{dm}^{-3}\right)$ segundo classificação de Trani \& Raij (1997). Com isso, a resposta obtida neste estudo quando se trabalhou com solo com teores muito baixos de potássio $\left(0,4 \mathrm{mmol}_{\mathrm{c}} \mathrm{dm}^{-3}\right)$ mostra que a espécie responde a doses de adubo superiores ao recomendado para o estado de São Paulo (Trani \& Raij, 1997).

A redução da produtividade observada no experimento, quando foi utilizada dose de $825 \mathrm{~kg} \mathrm{ha}^{-1}$ de $\mathrm{K}_{2} \mathrm{O}$, junto com determinada dose de $\mathrm{N}$, pode ter sido causada pelo efeito salino ao redor das raízes, comprometendo o seu crescimento e a absorção de água e nutrientes, efeito este observado em pimentão por Silva et al. (2001).

Quando foram avaliadas as doses crescentes de nitrogênio, para cada dose de potássio $\left(275,550\right.$ e $\left.825 \mathrm{~kg} \mathrm{ha}^{-1} \mathrm{~K}_{2} \mathrm{O}\right)$ foram obtidos efeitos quadráticos para produtividade (Figura 1B), pode-se estimar que as doses 286,91, 286,12 e $328,15 \mathrm{~kg} \mathrm{ha}^{-1}$ de $\mathrm{N}$ permitiram estimar as máximas produções de 333,88 , 344,62 e 344,73 g planta $^{-1}$ de inflorescência, respectivamente para as doses de 275,550 e $825 \mathrm{~kg} \mathrm{ha}^{-1}$ de $\mathrm{K}_{2} \mathrm{O}$.

Observou-se que as doses que proporcionaram máxima resposta variaram de 333 a $344 \mathrm{~kg} \mathrm{ha}^{-1}$ de nitrogênio e são superiores às recomendadas por Trani \& Raij (1997), que sugerem adubações variando de 75 a $260 \mathrm{~kg} \mathrm{ha}^{-1}$, para uma densidade de cerca de 25.000 plantas $\mathrm{ha}^{-1}$. Na literatura foram encontradas diversas doses recomendadas por vários autores: $196 \mathrm{~kg} \mathrm{ha}^{-1}$ (44.400 plantas ha-1) (Everaats, 1994), $250 \mathrm{~kg} \mathrm{ha}^{-1}(63.000$ plantas ha-1) (Kowalenko \& Hall, 1987), $265 \mathrm{~kg} \mathrm{ha}^{-1}$ (74.000 plantas ha-1), $300 \mathrm{~kg}$ ha $^{-1}$ (44.400 plantas ha ${ }^{-1}$ ) (Everaarts \& Willigen, 1999), $371 \mathrm{~kg} \mathrm{ha}^{-1}$ (148.000 plantas ha-1) (Kahn et al., 1991), 420 $\mathrm{kg} \mathrm{ha}^{-1}$ (77.000 plantas ha-1) (Zebarth et al., 1995$)$ e $315 \mathrm{~kg} \mathrm{ha}^{-1}$ (62.500 plantas $\mathrm{ha}^{-1}$ ) e $233 \mathrm{~kg} \mathrm{ha}^{-1}$ (25.000 plantas ha-1) (Schiavon Júnior, 2008).

Ao verificar a dose por planta aplicada pode-se notar que neste trabalho a resposta foi aproximadamente de 11 g planta ${ }^{-1}$ de nitrogênio e nos trabalhos expostos as doses variaram de 2,5 a 9,32 g planta ${ }^{-1}$ de nitrogênio.

Na dose de $450 \mathrm{~kg} \mathrm{ha}^{-1}$ de nitrogênio, pode-se verificar uma tendência de redução da produtividade que pode ter sido causada pelo excesso de nitrogênio. Nas inflorescências foram observadas pontuações amareladas, aparentando abortamento das flores.

Com relação à severidade da doença, foi observada maior retenção de folhas em plantas não inoculadas $(77,40 \%)$ quando comparadas com aquelas que foram inoculadas com $X$. campestris pv. campestris $(65,77 \%)$ (Tabela 3$)$. Esse resultado justifica as diferenças na produtividade da inflorescência, entre as plantas controles e as inoculadas, pois as folhas que permaneceram retidas contribuíram para a produção de fotoassimilados proporcionando aumento da biomassa da inflorescência.

Para a quantidade de folhas retidas, o potássio apresentou um efeito quadrático, sendo possível estimar o máximo de retenção das folhas na planta $(75 \%)$ na dose de $550 \mathrm{~kg} \mathrm{ha}^{-1}$ de $\mathrm{K}_{2} \mathrm{O}$ (Figura 2).

Observou-se que o aumento das doses de potássio e de nitrogênio apresentou efeito quadrático na área necrosada do caule e que as menores quantidades (54 e 52\%) foram obtidas nas doses de $800 \mathrm{~kg} \mathrm{ha}^{-1}$ de $\mathrm{K}_{2} \mathrm{O}$ e $283 \mathrm{~kg} \mathrm{ha}^{-1}$ de nitrogênio, respectivamente (Figura 3 ).

A dose de nitrogênio estimada para redução da área necrosada do caule foi um pouco menor que as estimadas para produtividade (286 a $328 \mathrm{~kg} \mathrm{ha}^{-1}$ ), mostrando que ao adubar com doses superiores à recomendada para produção pode reduzir a resistência à doença. Em estudo avaliando Pseudomonas marginalis e doses de nitrogênio em brócolis, Everaarts (1994) observou maior severidade da doença em plantas que receberam maior adubação nitrogenada.

Outro fato observado e já conhecido é que a deficiência de nitrogênio provoca subdesenvolvimento da planta, tornando-a menos vigorosa e, portanto, mais predisposta às doenças (Figura 3B). Esse fator é descrito por Huber (1994) para diversas doenças em hortaliças.

Quanto à adubação potássica, verifica-se que a dose de $800 \mathrm{~kg} \mathrm{ha}^{-1}$ de $\mathrm{K}_{2} \mathrm{O}$ reduziu a suscetibilidade (Figura 3A), mas está acima da estimada para máximas produções, que variaram de 541 a $751 \mathrm{~kg} \mathrm{ha}^{-1}$ de $\mathrm{K}_{2} \mathrm{O}$. Segundo Huber (1994), a suscetibilidade diminui da mesma forma que o crescimento da planta responde ao aumento do suprimento de potássio.

A nutrição mineral balanceada torna as plantas mais resistentes que as plantas com deficiência ou excesso nutricional (Zambolin \&Ventura, 1996). A influência da nutrição mineral na resistência é maior em cultivares moderadamente suscetíveis que em plantas altamente suscetíveis (Marscher, 1995). Santos et al. (2008) e Seabra et al. (2008) verificaram que no mercado existem opções de cultivares de brócolis com resistência ou moderada resistência à podridão negra, que podem contribuir no manejo produtivo, podendo reduzir perdas de produtividade causadas pela doença.

A adubação equilibrada consiste em suprir a necessidade especifica de cada nutriente exigido pela espécie. Para Peruch et al. (2006), o suposto equilíbrio nutricional dos cultivos orgânicos não foi suficiente para eliminar ou mesmo reduzir a severidade à podridão negra. Assim, os autores colocaram como hipótese que pode ter ocorrido um estresse nutricional, ou seja, é possível que nesse sistema de cultivo não esteja disponibilizando quantidades necessárias de nutrientes para o brócolis, já que a fertilização orgânica apresenta lenta liberação de nutrientes e as cultivares disponíveis no mercado são selecionadas para o sistema convencional de 
produção.

Levando em consideração a alta exigência nutricional da espécie propõe-se que uma adubação equilibrada para brócolis utilize $283 \mathrm{~kg} \mathrm{ha}^{-1}$ de $\mathrm{N}$ e 550 $\mathrm{kg} \mathrm{ha}^{-1}$ de $\mathrm{K}_{2} \mathrm{O}$ para obter maior produtividade de inflorescência e maior resistência à podridão negra.

\section{AGRADECIMENTOS}

Ao CNPQ pela concessão da bolsa e à FAPESP pelo auxílio financeiro.

\section{REFERÊNCIAS}

DZHALILOV FS; TIWARI RD. 1995. Soil and cabbage plant debris as infection sources of black rot. Archive für Phytopathologie und Pflanzenschutz 29: 383-387.

EVERAARTS AP. 1994. Nitrogen fertilization and head rot in broccoli. Netherlands Journal of Agricultural Science 42: 195-201.

EVERAARTS AP; WILLIGEN P. 1999. The effects of the rate and method of nitrogen application on nitrogen uptake and utilization by broccoli. Netherlands Journal of Agricultural Science 47: 201-214.

FERREIRA DF. 2000. Análises estatísticas por meio do Sisvar para Windows versão 4.0. In: 45a Reunião Anual da Região Brasileira da Sociedade internacional de Biometria. UFSCar, São Carlos-SP. p. 255-258.

FILGUEIRA FAR. 2008. Novo Manual de Olericultura: agrotecnologia moderna produção e comercialização de hortaliças. 3 ed. Viçosa: UFV. 421p.

HENZ GP; TAKATSUA; REIFSCHNEIDER FJB. 1988. Avaliação de métodos de inoculação de Xanthomonas campestris patovar campestris para detecção de fontes de resistência em brássicas. Fitopatologia Brasileira 13: 207210.

HOMA P; HAAG HP; SARRUGE JR. 1981. Deficiência de macronutrientes em couve- flor. In: HAAG HP; MINAMI K. Nutrição Mineral em Hortaliças. Campinas: Fundação Cargil. p.83-100.

HUBER DM. 1994. The influence of mineral nutrition on vegetable diseases. Horticultura Brasileira 12: 206-214.

LALLA JG; LAURA VA; RODRIGUES APDC; SEABRA JR S; SILVEIRA DS; ZAGO VH; DORNAS MF. 2010. Competição de cultivares de brócolos tipo cabeça única em Campo Grande. Horticultura Brasileira. 28: 360-363.

KAHN BA; AHILLING PG; BRUSEWITZ GH; McNEW RW. 1991. Force to shear the stalk, stalk diameter, and yield of broccoli in response to nitrogen fertilization and withinrow spacing. Journal of the American Society for Horticultural Science 116: 222-227.

KOWALENKO CG; HALL JW. 1987. Effects of nitrogen application on direct-seed Broccoli from a single harvest adjusted for maturity. Journal of the American Society for Horticultural Science 112: 9-13.

MAGNÍFICO V; LATTANZIO V; SARLI G. 1979. Growth and nutrient removal by broccoli. Journal of the American Society for Horticultural Science 104: 201-203.

MARSCHNER H. 1995. Mineral nutrition of higher plants. London: Academic Press. $889 \mathrm{p}$.

PECK NH; MacDONALD GE. 1986. Cauliflower, broccoli, and brussel sprouts responses to concentrated superphosphate and potassium chloride fertilization. Journal of the American Society for Horticultural Science 111: 195 201.

PERUCH LAM; MICHEREFF SJ; ARAÚJO IB. 2006. Levantamento da intensidade da alternariose e podridão negra em cultivos orgânicos de brássicas em Pernambuco e Santa Catarina. Horticultura Brasileira 24: 464-469.

RINCON L; SAEZ J; CRESPO JAP; LOPEZ MDG; PELLISER C. 1999. Crecimiento y absorcion de nutrientes del brocoli. Investigación Agraria: Producción $Y$ Protección Vegetal 14: 226-236.

SANTOS LA; BANDEIRA DA; SILVA JP; SILVEIRA EB; GOMES AMA; MARIANO RLR. 2008. Caracterização de isolados Xanthomonas campestris pv. Campestris de sistemas de produção orgânico e reação de brássicas à podridão-negra. Horticultura
Brasileira 26: 486-491.

SEABRAJÚNIOR S; PAIXÃO GS; MARINGONI AC; GOTO R; CAMARA RC. 2008. Reação de híbridos de brócolis 'tipo cabeça única' à podridão negra. Summa Phytopathologica 34: 76-77.

SCHIAVON JÚNIOR AA. 2008. Produtividade e qualidade de brócolis em função da adubação e espaçamento entre plantas. Jaboticabal: UNESP-FCAV. 67p (Dissertação mestrado).

SILVA JÚNIOR AA. 1986. Adubação mineral e orgânica em repolho 1ll. Qualidade comercial e ocorrência de $X$. campestris pv. Campestris. Horticultura Brasileira 4: 10-12.

SILVA MAG; BOARETTO AE; FERNANDES HG; BOARETTO R; MELO AMT; SCIVITTARO WBS. 2001. Características químicas de um latossolo adubado com uréia e cloreto de potássio em ambiente protegido. Scientia Agrícola 58: 561-563.

SILVEIRA RLVA; HIGASHI EN. 2003. Aspectos nutricionais envolvidos na ocorrência de doenças com ênfase para o eucalipto. Circular Técnica IPEF. N.200. p. 01-13. Disponível em http://www.rragroflorestal.com.br/documents/ ferrugem.pdf. Acesso em 10 de janeiro de 2011.

TAIZ L; ZEIGER E. 2009. Metabólitos secundários e defesa vegetal. In:__ Plant Physiology. Porto Alegre: Artmed. P342-372.

TRANI PE; RAIJ B. 1997. Hortaliças In: RAIJ B; CANTARELLAH; QUAGGIO JA; FURLANI AMC. Recomendações de adubação e calagem para o Estado de São Paulo. Campinas: IAC. 285p. (Boletim técnico 100).

YAMADA T. 2005. Nutrição x Doenças. Informações agronômicas 109: 8-13.

YAMADA T. 2004. Resistência de plantas às pragas e doenças. Informações agronômicas 108: 1-7.

ZAMBOLIN L; VENTURAJA. 1996. Resistência a doenças induzidas pela nutrição mineral das plantas. Informações Agronômicas 75: 1-16.

ZEBARTH BJ; BWEN PA; TOIVONEN MA. 1995. Influence of nitrogen fertilization on broccoli yield, nitrogen accumulation and apparent fertilizer-nitrogen recovery. Canadian Journal of Plant Science 75: 717725. 